\title{
Evaluation of the methoxy-X04 derivative BSC4090 for diagnosis of prodromal and early Alzheimer's disease from bioptic olfactory mucosa
}

\author{
Hannah Pellkofer ${ }^{1} \cdot$ Friedrich Ihler $^{2} \cdot$ Bernhard G. Weiss $^{2} \cdot$ Janina Trothe $^{3,4} \cdot$ Harindranath Kadavath $^{3} \cdot$ \\ Monika Chongtham ${ }^{3} \cdot$ Marcel Kunadt $^{5} \cdot$ Dietmar Riedel $^{6} \cdot$ Finn Lornsen $^{5} \cdot$ Petra Wilken $^{5} \cdot$ Claudia Bartels $^{5}$. \\ Sina Hirschel ${ }^{5}$. Sebastian G. Russo ${ }^{6}$. Elke Stransky ${ }^{7} \cdot$ Lutz Trojan $^{8}$ - Boris Schmidt ${ }^{9}$. Eckhardt Mandelkow ${ }^{10,11,12}$. \\ Markus Zweckstetter ${ }^{1,3,4} \cdot$ Martin Canis $^{2} \cdot$ Anja Schneider $^{10,13}$
}

Received: 6 July 2018 / Accepted: 3 November 2018 / Published online: 12 November 2018

(c) Springer-Verlag GmbH Germany, part of Springer Nature 2018

\begin{abstract}
Alzheimer's disease (AD) pathology precedes the onset of clinical symptoms by several decades. Thus, biomarkers are required to identify prodromal disease stages to allow for the early and effective treatment. The methoxy-X04-derivative BSC4090 is a fluorescent ligand which was designed to target neurofibrillary tangles in AD. BSC4090 staining was previously detected in post-mortem brains and olfactory mucosa derived from AD patients. We tested BSC4090 as a potential diagnostic marker of prodromal and early $\mathrm{AD}$ using olfactory mucosa biopsies from 12 individuals with $\mathrm{AD}, 13$ with mild cognitive impairment (MCI), and 10 cognitively normal (CN) controls. Receiver-operating curve analysis revealed areas under the curve of 0.78 for $\mathrm{AD}$ versus $\mathrm{CN}$ and of 0.86 for MCI due to $\mathrm{AD}$ versus MCI of other causes. BSC4090 labeling correlated significantly with cerebrospinal fluid levels of tau protein phosphorylated at T181. Using NMR spectroscopy, we find that BSC4090 binds to fibrillar and pre-fibrillar but not to monomeric tau. Thus, BSC4090 may be an interesting candidate to detect $\mathrm{AD}$ at the early disease stages.
\end{abstract}

Keywords Alzheimer's disease $\cdot$ Biomarker $\cdot$ Tau $\cdot$ Olfactory epithelia $\cdot$ Methoxy-X04

Electronic supplementary material The online version of this article (https://doi.org/10.1007/s00406-018-0955-6) contains supplementary material, which is available to authorized users.

Anja Schneider

anja.schneider@dzne.de

1 Department of Neurology, University Medical Center, Göttingen, Germany

2 Department of Otorhinolaryngology, Head and Neck Surgery, University Medical Center, Göttingen, Germany

3 German Center for Neurodegenerative Diseases, DZNE Göttingen, Göttingen, Germany

4 Max-Planck-Institute for Biophysical Chemistry, Göttingen, Germany

5 Department of Psychiatry and Psychotherapy, University Medical Center, Göttingen, Germany

6 Department of Anaesthesiology, University Medical Center, Göttingen, Germany

7 Department of Neurology, University Tübingen, Tübingen, Germany

\section{Abbreviations}

AD

AUC
Alzheimer's disease

Area under the curve
8 Department of Urology, University Medical Center, Göttingen, Germany

9 Clemens Schoepf-Institute of Organic Chemistry and Biochemistry, Technische Universität Darmstadt, Darmstadt, Germany

10 German Center for Neurodegenerative Diseases, DZNE Bonn, Sigmund-Freud-Str. 27, 53127 Bonn, Germany

11 Center of Advanced European Studies and Research, 53175 Bonn, Germany

12 Max Planck Institute for Metabolism Research, 22607 Hamburg, Germany

13 Department of Neurodegenerative Diseases and Geriatric Psychiatry, University Bonn, 53127 Bonn, Germany 


$\begin{array}{ll}\text { CERAD } & \begin{array}{l}\text { Consortium to Establish a Registry } \\ \text { for Alzheimer's Disease } \\ \text { CN }\end{array} \\ \text { CSF } & \text { Cerebritively normal } \\ \text { FCS } & \text { Fetal calf serum } \\ \text { FPLC } & \text { Fast protein liquid chromatography } \\ \text { HSQC } & \text { Heteronuclear single quantum } \\ & \text { coherence } \\ \text { MMSE } & \text { Mini-mental state examination } \\ \text { NIA-AA } & \text { National Institute on Aging-Alzhei- } \\ & \text { mer's Association } \\ \text { NFT } & \text { Neurofibrillary Tangles } \\ \text { NINCDS-ADRDA } & \text { National Institute of Neurological } \\ & \text { and Communicative Disorders and } \\ & \text { Stroke and the Alzheimer's Disease } \\ & \text { and Related Disorders Association } \\ \text { NMR } & \text { Nuclear magnetic resonance } \\ \text { OE } & \text { Olfactory epithelia } \\ \text { PET } & \text { Positron emission tomography } \\ \text { ROC } & \text { Receiver-operating curve } \\ \text { SUVR } & \text { Standard uptake value ratio } \\ \text { STD } & \text { Saturation-transfer difference }\end{array}$

\section{Introduction}

Alzheimer's disease (AD) is characterized by deposition of amyloid plaques and neurofibrillary tangles (NFTs) composed of aggregated tau protein [14, 21, 38]. AD pathology begins decades before clinical symptoms emerge [7, $23,35]$. Therefore, therapeutic interventions to prevent or slow down disease progression should start at the earliest possible time point. Thus, there is an urgent need for novel biomarkers to detect $\mathrm{AD}$ pathology in the prodromal or even preclinical disease stage. Whereas substantial progress has been achieved with PET tracers detecting amyloid plaques, biomarkers indicating tau pathology in $\mathrm{AD}$ are restricted to high levels of phosphorylated tau in cerebrospinal fluid (CSF).

Olfactory impairment is observed in many neurodegenerative disorders including $\mathrm{AD}[6,16]$. It precedes the onset of clinical symptoms by many years, constitutes a risk factor for mild cognitive impairment (MCI), and is associated with rapid cognitive decline $[15,40]$ and a high burden of tau pathology. AD pathology has been observed in the olfactory bulb but also in peripheral olfactory epithelia (OE) [4]. For example, tau immunoreactive dystrophic neurites [2, 37], pathologically phosphorylated tau and fibrillar tau deposits $[3,27,43]$ were detected in post-mortem and bioptic OE tissue [36] derived from AD patients. However, the sensitivity to detect $\mathrm{AD}$ and the specificity to distinguish $\mathrm{AD}$ from controls were low. Of note, immunoreactivity against phosphorylated tau was absent in OE biopsies from five patients with mild and moderate $\mathrm{AD}$ and may thus emerge only at later disease stages [22].

The fluorescent methoxy-XO4 derivative BSC4090 binds to NFTs in human AD brains with high affinity and specificity and colocalizes with NFT-tau phosphorylated at S202/ T205 (AT8) [13]. BSC4090 also labels post-mortem OE from neuropathologically confirmed AD patients [13].

$\mathrm{We}$, therefore, evaluated the potential of BSC4090 to detect tau pathology in OE biopsies of individuals in the early and prodromal AD stages.

\section{Materials and methods}

\section{Standard protocol approvals, registrations, and patient consents}

After written informed consent, nasal mucosa biopsies were collected at the University Medical Center Göttingen, Germany. Between 2012 and 2014, 12 subjects with a diagnosis of $\mathrm{AD}, 13$ with a diagnosis of MCI, and 10 cognitively normal controls $(\mathrm{CN})$ underwent biopsy (IRB approval 25/2/12 by the local ethics committee). All procedures involving human participants were in accordance with the $1964 \mathrm{Hel}-$ sinki declaration and its later amendments.

\section{CSF collection}

Cerebrospinal fluid (CSF) samples were obtained from nine subjects with $\mathrm{AD}, 13$ with $\mathrm{MCI}$, and two from the $\mathrm{CN}$ group. All CSF samples were retrieved by lumbar puncture, collected in polypropylene tubes, and centrifuged at $2000 \times g$ for $10 \mathrm{~min}$ at room temperature. Supernatants were aliquoted and frozen at $-80{ }^{\circ} \mathrm{C}$ within $30 \mathrm{~min}$ of the procedure's completion.

\section{Assessments}

AD patients fulfilled the National Institute of Neurological and Communicative Disorders and Stroke and the Alzheimer's Disease and Related Disorders Association (NINCDSADRDA) criteria for probable AD. MCI diagnosis was based on the recommendations from the National Institute on Aging-Alzheimer's Association workgroups on diagnostic guidelines for Alzheimer's disease (NIA-AA) [1] According to the NIA-AA criteria, a diagnosis of MCI due to $\mathrm{AD}$ was given based on the presence of AD biomarkers in CSF. Subjects fulfilling the clinical and neuropsychological criteria of MCI were classified as MCI due to $\mathrm{AD}$ if either CSF tau or CSF amyloid- $\beta 42$ or both were in the pathological range [1] with CSF tau levels above and/or CSF amyloid- $\beta 42$ concentrations below the cutoff. Cut-off 
values were based on Dumurigier et al. [17], with $568 \mathrm{pg} / \mathrm{mL}$ as cutoff for CSF amyloid- $\beta 42$ and $307 \mathrm{pg} / \mathrm{mL}$ for CSF tau.

All participants with $\mathrm{AD}$ or MCI underwent brain MRI or cranial computer tomography imaging to rule out the other causes of dementia or cognitive impairment.

\section{Neuropsychological assessments}

$\mathrm{AD}, \mathrm{MCI}$, and $\mathrm{CN}$ control subjects were assessed with the Consortium to establish a registry for Alzheimer's disease (CERAD) Plus neuropsychological test battery [18, 32]. MCI was defined as the combination of memory complaints plus a performance of 1-1.5 standard deviations below the age norm on at least one cognitive domain, while activities of daily living were preserved [41]. CN controls were recruited from the departments of urology and otorhinolaryngology, head and neck surgery at the University Medical Center Göttingen, and included patients with prostate and bladder cancer or chronic sinusitis who underwent surgery with or without spinal anesthesia. Specimens of cognitively normal controls were collected from the endonasal mucosa in the olfactory area where endoscopically no sign of chronic sinusitis was detected. Patients recruited from the department of urology had no history of nasal pathology. Patients recruited from the department of otorhinolaryngology, head and neck surgery had mild chronic sinusitis without nasal polyps affecting single sinuses due to anatomical abnormalities (e.g., deviated nasal septum narrowing the ostiomeatal complex). This had no impact on the mucosa in the region where specimens have been collected. None of the $\mathrm{CN}$ controls fulfilled criteria for MCI or dementia nor suffered from a neurodegenerative disease. For patients' characterization, please refer to supplementary Table $\mathrm{S} 1$.

\section{Sniffin sticks test}

Olfactory function was evaluated using the Sniffin Sticks test battery consisting of a discrimination and an identification test [10]. For odor presentation, the cap of the pen was removed by the investigator for approximately $3 \mathrm{~s}$ and the odorized tip was placed approximately $2 \mathrm{~cm}$ in front of the subject's nostrils. All olfactory tests were performed bilaterally.

\section{Biopsies of nasal mucosa}

Specimens were taken randomly or at the side that was most easily accessible if a deviated nasal led to a narrow septum on one side. Local anesthesia was obtained by placing a cotton wool with Tetracaine hydrochloride $2 \%$ and Xylometazoline hydrochloride $0.1 \%$ in the nasal cavity above the middle turbinate. Consequently, an angled Blakesley forceps was introduced through the naris and advanced between the septum and middle turbinate. The cutting edge was pressed against the olfactory mucosa and withdrawn with the mucosal specimen. The specimens were immediately placed on $4 \%$ paraformaldehyde in PBS for $1-2$ days at $4{ }^{\circ} \mathrm{C}$.

\section{Immunohistochemistry and BSC4090 labeling}

Fixed tissue was embedded in paraffin and serially sectioned at $1 \propto \mathrm{m}$ intervals. Sections were deparaffinised and boiled for $30 \mathrm{~min}$ in $10 \mathrm{mM}$ citrate buffer (pH 6.0). After $10 \mathrm{~min}$ incubation in $3 \% \mathrm{H}_{2} \mathrm{O}_{2}$ and blocking for $30 \mathrm{~min}$ in $10 \%$ fetal calf serum (FCS), probes were stained with antibodies against hyperphosphorylated tau (AT8, 1:1000, Thermo Scientific; Germany). Sections were treated with biotin-conjugated anti-mouse immunoglobulins (Abcam) which were visualized by a horseradish peroxidase-diaminobenzidine (Sigma-Aldrich, Germany) reaction. All sections were additionally stained with hemalaun following standard protocols.

The divinylpyridazine derivate 4-((1E)-2-(6-(4(dimethylamino)styryl)pyrimidin-4-yl)vinyl)- $N, N$-dimethylbenzylamin BSC4090 was synthesized and used for staining of adjacent sections as described previously [12]. Briefly, deparaffinized sections were incubated for $10 \mathrm{~min}$ at room temperature in $1 \mathrm{~g} / \mathrm{l} \mathrm{BSC4090} \mathrm{dissolved} \mathrm{in} \mathrm{metha-}$ nol. Sections were then washed for $1 \mathrm{~min}$ in distilled water and incubated in $1 \%$ acetic acid for $15 \mathrm{~min}$ at room temperature, followed by two 1 min washing steps in distilled water. Bowman glands were identified by their characteristic morphology.

\section{Microscopy}

Fluorescence images were acquired with a confocal laser scanning microscope (Leica SP2, Leica, Germany). BSC4090 was excited at $488 \mathrm{~nm}$ with identical laser settings for all the samples. DAB stained sections and images for quantitative analysis of BSC4090 were acquired at an inverse epifluorescence microscope (MEA53100 Ti-E, Nikon, Düsseldorf, Germany). Large-scale images to cover the complete section were composed by stitching single images, using the NIS Elements AR software (Nikon, Düsseldorf, Germany).

\section{CSF analysis}

The analysis of CSF biomarkers was performed by solidphase enzyme immunoassays for amyloid- $\beta_{1-42}$, total tau, and p-tau 181 (Innotest, Fujirebio Europe).

\section{Data analysis}

Data analysis was performed by a rater blinded to the diagnosis. For all BSC4090 labeled sections, adjacent sections were stained with haemalaun to verify the presence 
of olfactory Bowman glands. At least three different sections were analyzed per participant. The BSC4090 ratio was calculated as the number of BSC4090 positive to the total number of Bowman gland cells from each section.

\section{NMR spectroscopy}

\section{Proteins and chemicals}

pNG2 plasmid with inserted coding sequences of the fulllength tau isoform hTau40 and tau construct K18 (amino acids 244-372) were transformed into E. coli strain BL21(DE3) (Merck-Novagen, Darmstadt) [20]. Proteins were purified as described previously [5]. Uniformly ${ }^{15} \mathrm{~N}$ enriched htau40 sample was prepared by growing $E$. coli bacteria in minimal medium containing $1 \mathrm{~g} / \mathrm{l}$ of ${ }^{15} \mathrm{NH}_{4} \mathrm{Cl}$ according to the established protocols [33, 34]. The compound BSC4090 was synthesized in-house [13].

\section{Protein aggregation}

Aggregation of K18 was induced by incubating $10 \mu \mathrm{M}$ soluble K18 and $1 \mathrm{mM}$ DTT by adding the anionic cofactor heparin (Sigma, Germany) in $50 \mathrm{mM}$ sodium phosphate buffer, pH 6.8 at $37^{\circ} \mathrm{C}$, with a molar ratio of $\mathrm{K} 18$ to heparin of $4: 1$. The solution was incubated at $37{ }^{\circ} \mathrm{C}$ before NMR experiments for $30 \mathrm{~min}$ and continued at $25^{\circ} \mathrm{C}$ for $\sim 3$ days during the course of NMR experiment. A parallel sample was used to monitor the formation of aggregates by electron microscopy.

\section{Electron microscopy}

Samples containing aggregates of K18 were diluted, deposited onto carbon-coated copper mesh grids and negatively stained with $1 \%(\mathrm{w} / \mathrm{v})$ uranyl acetate. Samples were examined using a Philips CM 120 BioTwin transmission electron microscope (FEI, Eindhoven, The Netherlands). Pictures were taken with a TemCam F416 CMOS camera (TVIPS, Gauting, Germany).

\section{NMR spectroscopy}

BSC4090 was dissolved in DMSO-D6 at a concentration of $10 \mathrm{mM}$. NMR measurements were performed in $50 \mathrm{mM}$ sodium phosphate buffer, $\mathrm{pH} 6.8,10 \% \mathrm{D}_{2} \mathrm{O}$ and $3 \%$ DMSOD6. Two-dimensional (2D) ${ }^{1} \mathrm{H}^{15} \mathrm{~N}$ heteronuclear single quantum coherence (HSQC) experiments $[8,9,11]$ of tau in the presence and absence of the compound BSc4090 were recorded at $5{ }^{\circ} \mathrm{C}$ on a $700 \mathrm{MHz}$ Bruker spectrometer equipped with a cryoprobe prodigy. The spectra were processed using Topspin 3.1 (Bruker) and analyzed using
Sparky 3.114 (T. D. Goddard and D. G. Kneller, http://www. cgl.ucsf.edu/home/sparky).

To probe the interaction of the ligand BSC4090 with the aggregates/fibrils of K18 (tau244-372), saturation-transfer difference (STD) NMR spectra [25, 28] of BSC4090 with and without $\mathrm{K} 18$ aggregates were recorded. The concentrations of the ligand and $\mathrm{K} 18$ were $150 \mu \mathrm{M}$ and $10 \mu \mathrm{M}$, respectively. 1D STD NMR spectra were recorded at $25^{\circ} \mathrm{C}$ on a $600 \mathrm{MHz}$ spectrometer equipped with a cryoprobe using a series of 40 equally spaced $50 \mathrm{~ms}$ Gaussian-shaped pulses for saturation of the protein, with a total saturation time of $1.5 \mathrm{~s}$. To saturate the protein protons, on- and off-resonance frequencies were set to $-0.5 \mathrm{ppm}$ and $60 \mathrm{ppm}$, respectively.

\section{Results}

\section{Collection of olfactory mucosa biopsies}

To test the potential of BSC4090 as a diagnostic antemortem marker of AD pathology, we obtained biopsies from study participants diagnosed with either $\mathrm{AD}, \mathrm{MCI}$ or from $\mathrm{CN}$ controls. The cognitive state of each individual was assessed by the CERAD Plus test battery [32].

Intranasal biopsies were well tolerated. No adverse events such as excessive bleeding, pain, or olfactory nerve injury were observed. Tissue specimens were cut into $1 \mu \mathrm{m}$ sections and every tenth section was labeled with BSC4090. Sections with less than 5 Bowman glands were excluded from analysis. Tissue volumes of at least $2 \mathrm{~mm}$ diameter and correct location of the biopsy from the nasal cavity above the middle turbinate allowed the quantification of gland pathology and were obtained after training of the biopsy procedure. An overview of demographic data including age, gender, and MMSE is given in Supplementary Table S1.

\section{BSC4090 labeling of mucosal biopsies distinguishes between $A D$ and $C N$ controls}

Evaluation of BSC4090 labeling was carried out by a rater blinded to the diagnosis. All 12 mild AD cases showed strong BSC4090 labeling of Bowman glands (Fig. 1a, b). Frequently, BSC4090 staining was restricted to several, mostly adjacent cells or single cells within Bowman glands. BSC4090 labeling was mainly homogenous or granular but never fibrillar. This staining pattern is similar to that described for post-mortem $\mathrm{AD}$ OE $[12,13]$.

BSC4090 staining was also detected in eight out of ten $\mathrm{CN}$ controls, albeit to a markedly lower extent (Fig. 1c). We next quantified the ratio of BSC4090 positive to the total number of Bowman gland cells from at least three different sections per biopsy (Fig. 1d). BSC4090 ratios were significantly higher in the AD group as compared to 
Fig. 1 BSC4090 distinguishes between Alzheimer tissue (AD) and controls $(\mathrm{CN})$. a Overview of BSC4090 labeling in olfactory mucosa of bioptic AD OE tissue. Scale bar 200 $\mu \mathrm{m}$. b Higher magnification of BSC4090 positive Bowman glands in olfactory mucosa of bioptic AD OE tissue. Scale bar $70 \mu \mathrm{m}$. c No BSC4090 labeling in a biopsy of a cognitively normal $(\mathrm{CN})$ control (CSF biomarker negative). Scale bar $70 \mu \mathrm{m}$. d Box blot of BSC4090 ratios (=BSC4090 positive OE cells versus all $\mathrm{OE}$ cells) of $\mathrm{AD}$ and $\mathrm{CN}$ controls; $p=0.002$. e ROC curve analysis was performed to evaluate the diagnostic performance of BSC4090 ratios to distinguish $\mathrm{AD}$ from $\mathrm{CN}$ controls (area under the curve $\mathrm{AUC}=0.778, p=0.0125$ ) (a) BSC4090, biopsy, overview

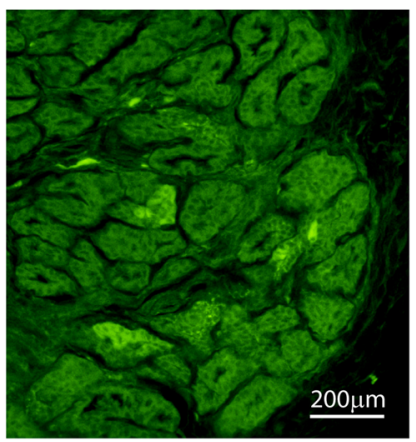

(b) BSC4090, biopsy, AD OE

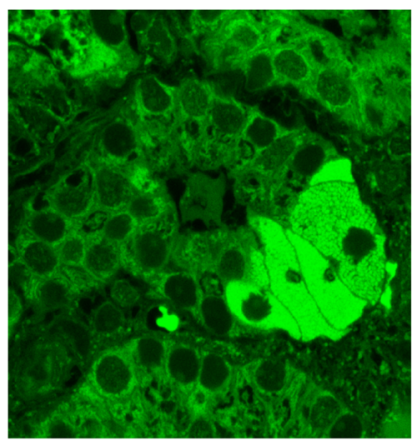

(c) BSC4090, biopsy, CN OE

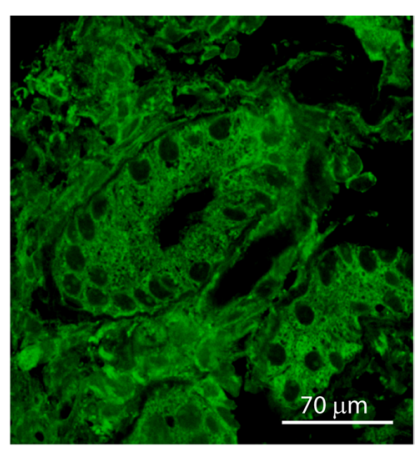

(d) Box plot $A D / C N$

$A D$

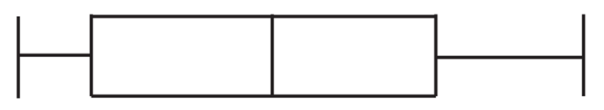

CN
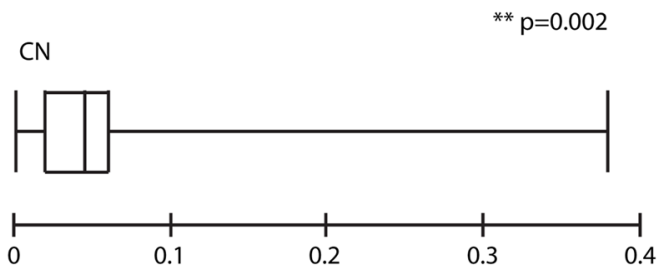

(e) ROC curve analysis $\mathrm{BSC} 4090$ ratios, $\mathrm{AD}$ versus $\mathrm{CN}$

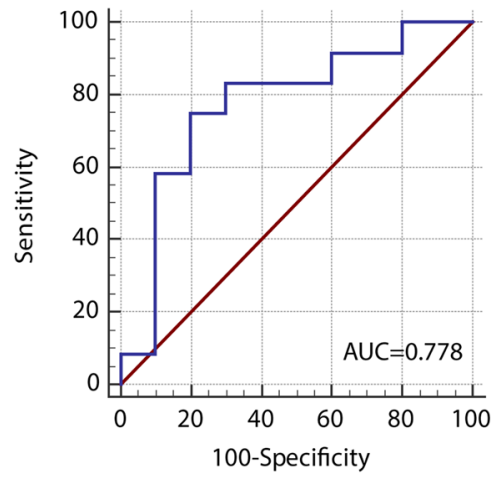

the CN group (AD: mean 0.18, $n=12 ; \mathrm{CN}$ : mean 0.073, $n=10, p=0.002$; Mann-Whitney $U$ test) (Fig. 1d). Receiver-operating curve (ROC) analysis revealed that the BSC4090 ratio distinguishes between AD and CN controls with $75 \%$ sensitivity and a specificity of $80 \%$. The area under the curve (AUC) was 0.778 ( $p=0.0125$ ) (Fig. 1e). Of note, one participant from the $\mathrm{CN}$ group reached a BSC4090 ratio of 0.377 far above the mean value of this group (0.076). It is tempting to speculate that this finding may indicate a preclinical stage of AD, although CSF data were not available for this participant to further support this notion.

\section{$\mathrm{MCl}$ due to AD is detected by BSC4090 staining}

Based on CSF amyloid- $\beta 42$ and tau levels, we divided the MCI group into MCI due to $\mathrm{AD}(n=6)$ and MCI with other causes $(n=7)$ (Fig. 2a, b). The BSC4090 ratio in the MCI due to AD group was significantly higher than in the MCI with other causes' group (MCI due to AD, mean 0.158; MCI other causes, mean 0.060, $p=0.0385$; Mann-Whitney $U$ test) (Fig. 2b). Of note, the BSC4090 ratios for MCI with other causes were similar to those of the $\mathrm{CN}$ group (MCI other causes, mean 0.060, compared to $\mathrm{CN}$, mean 0.073 , $p=0.928$, Mann-Whitney $U$ test). BSC4090 ratios detected 
(a) BSC4090, biopsy, MCI-AD

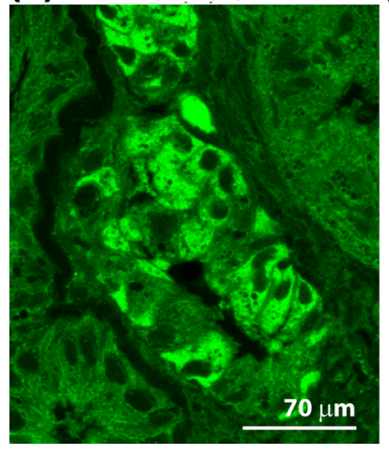

(b)

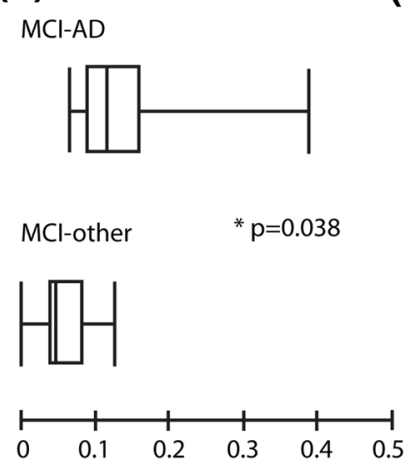

(c) ROC curve analysis MCI-AD/MCI-other

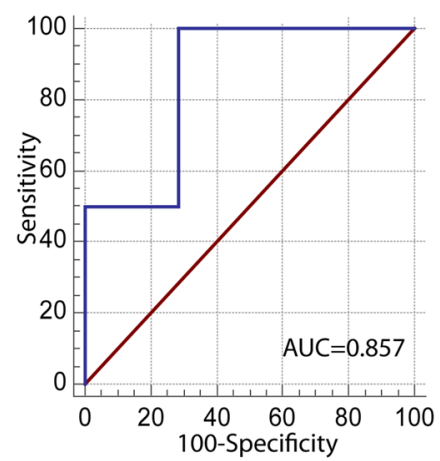

(e) ROC curve analysis combined groups

$A D+M C l-A D$
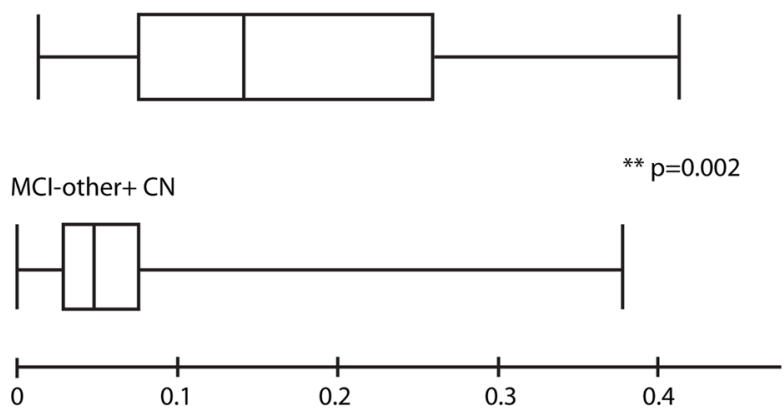

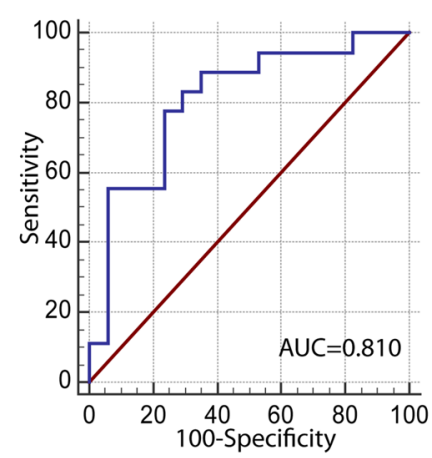

Fig. 2 BSC4090 distinguishes between MCI due to AD and MCI caused by other reasons. a BSC4090 staining of Bowman glands in olfactory mucosa biopsies of participants with MCI due to AD. Scale bar $70 \mu \mathrm{m}$. b Box blot analysis of BSC4090 ratios of olfactory mucosa biopsies of participants with MCI due to AD (upper bar) versus MCI caused by other reasons (lower bar); $p=0.038$. c ROC curve of bioptic olfactory mucosa BSC4090 ratios to distinguish MCI due to AD from MCI caused by other reasons (AUC $0.857, p=0.0015$ ). d Boxplot of BSC4090 ratios in olfactory mucosa biopsies of AD and MCI due to AD (AD pathology group) compared to the MCI caused by other reasons and $\mathrm{CN}$ group (control group) $(p=0.002)$. e ROC analysis of BSC4090 ratios to distinguish between AD and MCI due to $\mathrm{AD}$ (AD pathology group) compared to the MCI caused by other reasons and $\mathrm{CN}$ group (control group)(AUC $0.81, p<0.0001$ )
AD due to MCI with a sensitivity of $100 \%$ and distinguished it from MCI with other causes with a specificity of $71.4 \%$. ROC analysis revealed an AUC value of $0.857(p=0.0015)$ (Fig. 2c). We then combined $\mathrm{AD}$ and $\mathrm{MCI}$ due to $\mathrm{AD}$ into an "AD pathology group" and MCI with other causes and $\mathrm{CN}$ into a joint "control group". Figure $2 \mathrm{~d}$ shows box plots of these two groups with a mean BSC4090 ratio of 0.172 in the "AD pathology group" and 0.068 in the "control group" ( $p=0.002$, Mann-Whitney $U$ test) (Fig. 2d). The sensitivity of BSC4090 ratios to detect AD pathology amounted to $77.8 \%$ and the specificity was $76.5 \%$. ROC analysis revealed an AUC of $0.810(p<0.0001)$ (Fig. 2e).

\section{BSC4090 ratio correlates with CSF levels of ptau 181}

We next tested whether the percentage of BSC4090 positive $\mathrm{OE}$ cells increases with disease progression. To this end, we correlated BSC4090 ratios to CSF levels of tau as a marker of neuronal injury and tau phosphorylated at T181 (ptau181) as a more specific measure of AD tau pathology [19]. However, we did not observe a significant correlation between CSF levels of total tau and BSC4090 ratios (Fig. 3a). In contrast, CSF concentrations of ptau 181 were positively correlated with the BSC4090 ratio over all diagnostic groups (Pearson correlation $r=0.438846$, $p=0.0466, n=21$ ) (Fig. 3b). BSC4090 ratios did not correlate with CSF amyloid $\beta-42$ concentrations (Pearson correlation $0.20, p=0.35, n=22$ ) (Fig. 3c) nor with Mini-Mental State Examination (MMSE) scores (Pearson correlation $-0.22, p=0.29, n=35$, all diagnostic groups) (Fig. 2d). Pearson correlation between MMSE and BSC4090 ratios amounted to -0.07 in the combined $\mathrm{AD}+\mathrm{MCI}$ due to $\mathrm{AD}$ group (n.s., $n=17$, data not shown) and to -0.05 in the $\mathrm{AD}$ group (n.s., $n=11$, data not shown). MMSE scores may not accurately reflect cognitive impairment in MCI due to ceiling effects. Unfortunately, a possible correlation of CERAD test battery subscores with BSC4090 ratios could not be calculated due to the low number of subjects in the MCI due to $\mathrm{AD}$ group $(n=6)$ and bottom effects in the AD group (data not shown). 
(a) Correlation of CSF tau and BSC4090 ratios

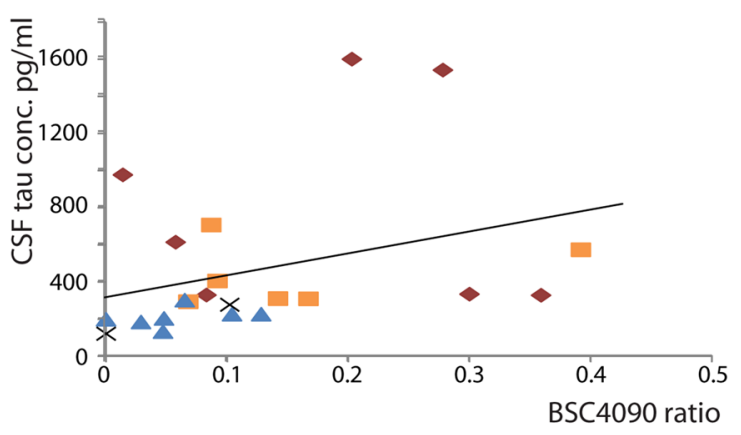

(c) Correlation of CSF amyloid- $\beta 42$ and BSC4090 ratios

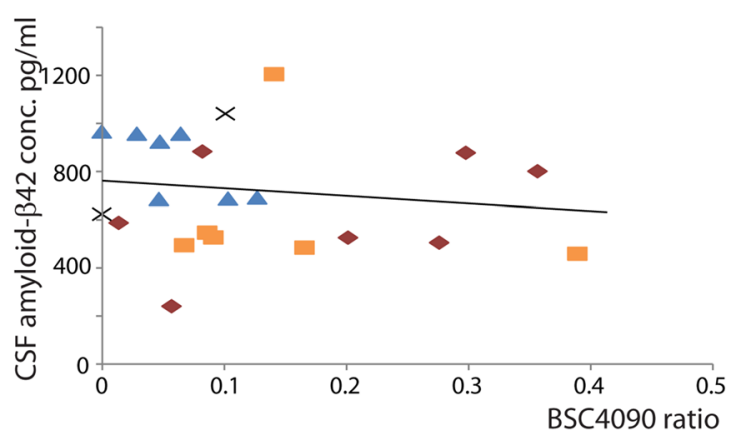

(e) Correlation of sniffin sticks scores and BSC4090 ratios

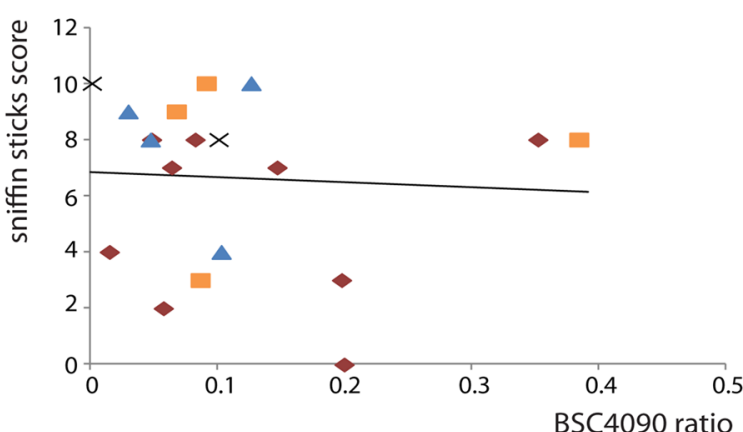

(b) Correlation of CSF p-tau181 and BSC4090 ratios

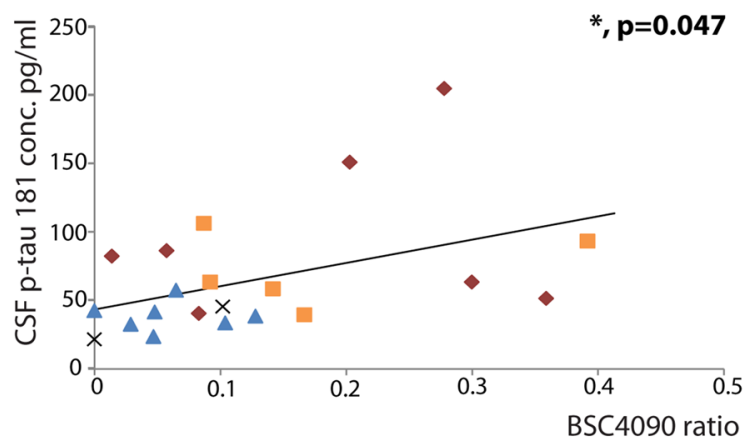

(d) Correlation of MMSE and BSC4090 ratios

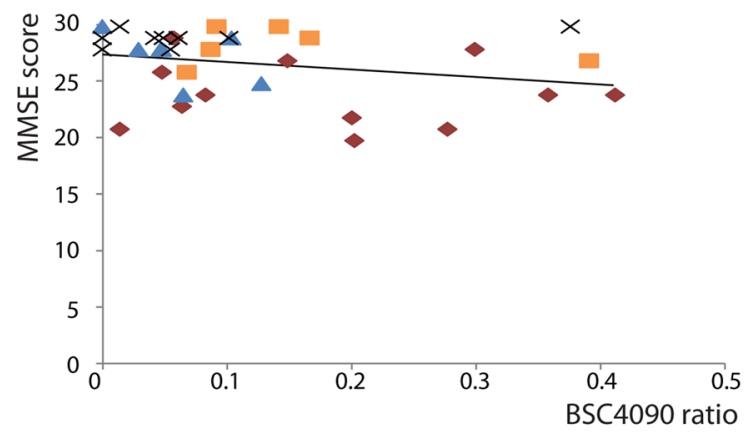

Fig. 3 BSC4090 ratios correlate with CSF concentrations of p-tau 181. a No correlation of CSF tau concentrations with corresponding BSC4090 ratios in olfactory mucosa biopsies. Pearson correlation coefficient $r=0.333, p=0.129, n=22$ across all diagnostic groups for which CSF was available. b BSC4090 ratio of olfactory mucosa biopsies blotted against the corresponding CSF concentration of tau phosphorylated at T181, $n=21$ across all diagnostic groups for which CSF was available. Pearson correlation coefficient $r=0.439$, $p=0.0466$. $\mathbf{c}$ No correlation of CSF amyloid- $\beta 42$ concentrations and

Interestingly, BSC4090 ratios did also not correlate with olfactory dysfunction as quantified by the sniffin sticks test (Pearson correlation $-0.088, p=0.72, n=19$ ) (Fig. 3e), although it cannot be excluded that olfactory dysfunction in the $\mathrm{AD}$ group rather reflects cognitive impairment than
BSC4090 ratios. Pearson correlation coefficient $r=0.333, p=0.129$, $n=22$ across all diagnostic groups for which CSF was available. $\mathbf{d}$ No correlation between MMSE scores and BSC4090 ratios. Pearson correlation coefficient $r=-0.271, p=0.117 . n=35$ across all diagnostic groups for which MMSE scores were available. e No correlation of sniffin sticks scores with BSC4090 ratios. Pearson correlation coefficient $r=0.063, p=0.798$. $n=19$ across all diagnostic groups for which sniffin sticks scores were available

the ability to detect the odor. Importantly, BSC4090 ratios did not significantly correlate with age in the joint $\mathrm{CN}$ and $\mathrm{MCI}$ non $\mathrm{AD}$ group, indicating that age per se is not the reason for the higher values observed in the AD and MCI due to $\mathrm{AD}$ groups (data not shown). 
(a)

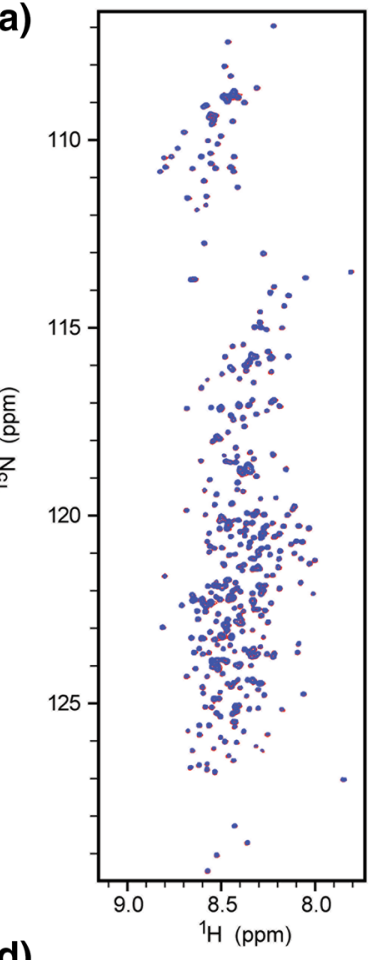

(d)

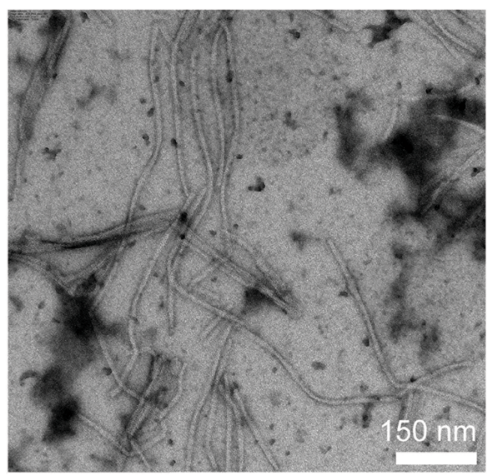

(f)

K18 fibrils+BSC4090 STD

K18 fibrils STD

K18 monomers STD

BSC4090 STD
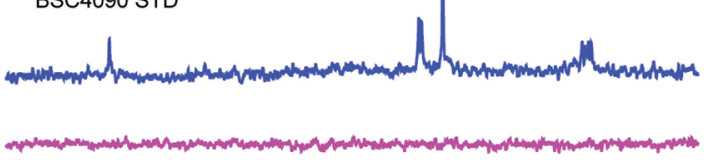

com

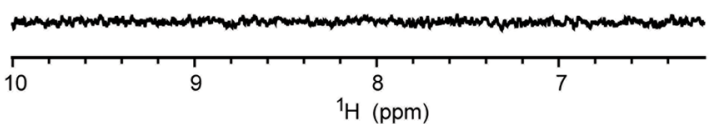

(c)

(e)

(g)
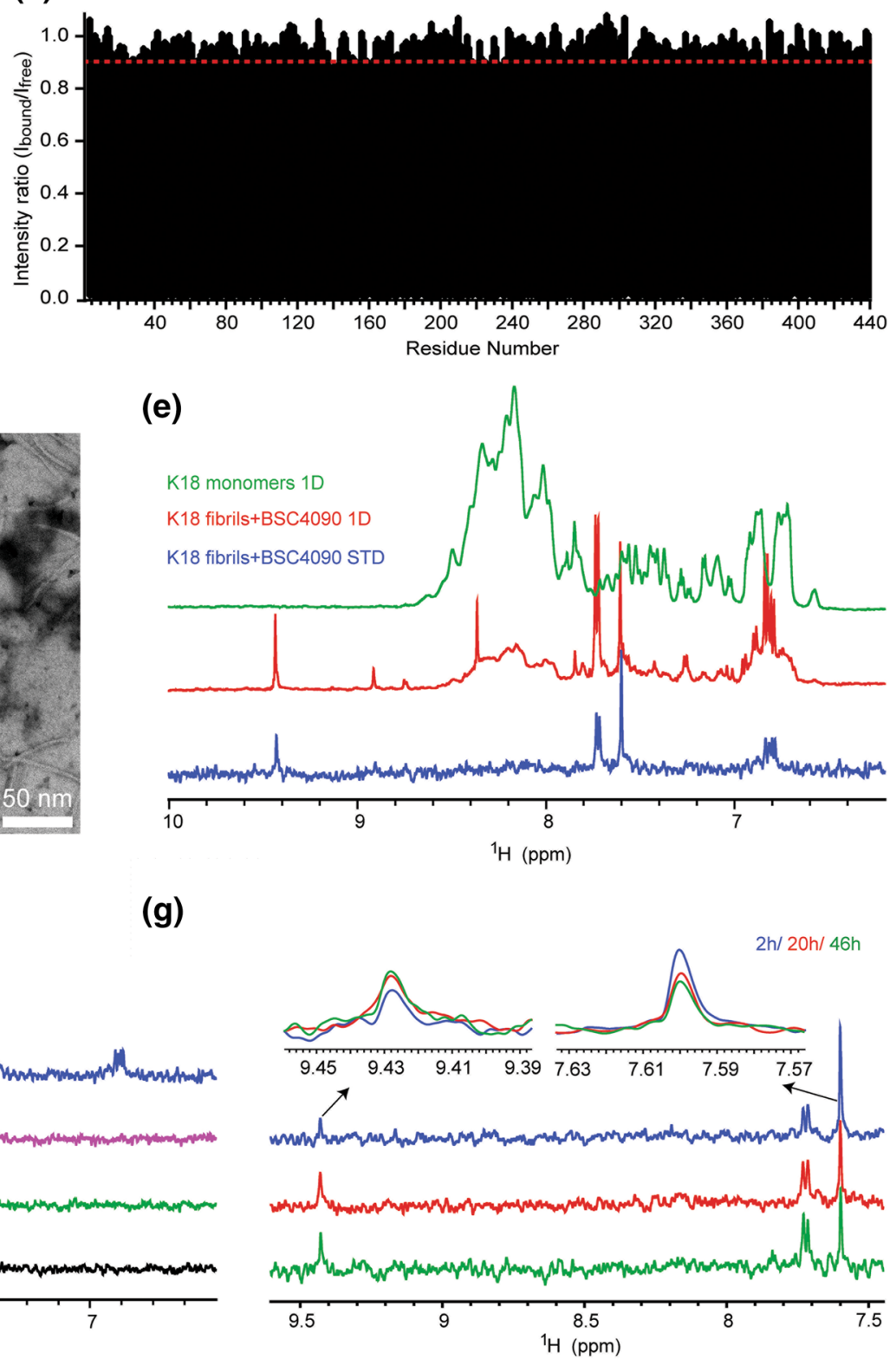
4Fig. 4 BSC4090 binds to fibrillar and pre-fibrillar tau. a, b Twodimensional ${ }^{1} \mathrm{H}_{-}{ }^{15} \mathrm{~N}$ HSQC NMR and one-dimensional ${ }^{1} \mathrm{H}$ NMR spectra of full-length, 4-repeat tau (htau40) with and without BSC4090. An overview on recombinant tau constructs is given in suppl. Fig. S1. Htau40 spectra are depicted in red and htau40 with BSC4090 in blue. Gray arrows indicate ${ }^{1} \mathrm{H}$ NMR signals originating from BSC4090. c NMR signal intensity ratios of cross peaks in the two-dimensional ${ }^{1} \mathrm{H}^{15}{ }^{15}$ HSQC NMR spectra of htau40 with and without BSC4090 were above 0.9 (dashed red line). d Electron micrograph (EM) of K18 fibrils generated by co-incubation of monomeric K18 with heparin. Scale bar $150 \mathrm{~nm}$. e Identification of the protons in BSC4090 involved in binding with K18 fibrils. Comparison of the 1D NMR spectrum of K18 monomers (green) with the 1D (red) and STD NMR spectra (blue) of K18 fibrils in the presence of BSC4090. f Comparison of STD NMR spectra of K18 fibrils with and without BSC4090. Blue: K18 fibrils BSC4090 STD, magenta: K18 fibrils STD, green: K18 monomers STD, black: BSC4090 STD. g Comparison of STD NMR spectra during fibril formation. K18 was incubated with heparin for $2 \mathrm{~h}$ (blue), $20 \mathrm{~h}$ (red), and $46 \mathrm{~h}$ (green). Fibril formation was tested by EM, which detected no fibrillar tau after $2 \mathrm{~h}$ in contrast to abundant fibril formation after $46 \mathrm{~h}$ (suppl. Fig. S1). STD NMR spectra indicate that BSC4090 binds to K18 coincubated with heparin for $2 \mathrm{~h}$, e.g., in the absence of fibrils, whereas BSC4090 does not bind to K18 monomers (f)

\section{BSC4090 binds to pre-fibrillar and fibrillar tau}

Since the BSC4090 staining pattern in Bowman glands did not resemble fibrillar morphology, we wondered whether BSC4090 would detect pre-fibrillar tau. Towards this end, we performed NMR spectroscopy of recombinant full-length tau (htau40, suppl. Fig. S1) with and without BSC4090. ${ }^{1} \mathrm{H}-$ ${ }^{15} \mathrm{~N}$ HSQC NMR spectra indicated that BSC4090 does not bind monomeric tau (Fig. 4a-c). In contrast, STD spectra of tau fibrils, which were assembled from the four repeat region of recombinant human tau (K18, suppl. Fig. S1) in vitro and either measured in the absence or presence of BSC4090, suggested the binding of BSC4090 to fibrillar tau (Fig. $4 \mathrm{~d}-\mathrm{g}$ ). The observed STD signals arise only from the protons of BSC4090 involved in binding (Fig. 4f). Comparison of STD NMR spectra of K18 measured in the presence of BSC4090 at different time points during in vitro aggregation revealed no major differences between K18 incubated for 2, 20 or $46 \mathrm{~h}$ (Fig. 4g). As shown by electron microscopy, $\mathrm{K} 18$ readily formed filaments after $46 \mathrm{~h}$, whereas no filamentous structures were detectable after $2 \mathrm{~h}$ of incubation (suppl. Fig. S2). This indicates that BSC4090 also binds pre-fibrillar intermediates of tau in addition to fibrillar tau aggregates.

\section{Discussion}

Here, we find that the methoxy-X04 derivative BSC4090 detects tau pathology in OE biopsies already in the early disease stages such as MCI. In our pilot study, the ratio of BSC4090 positive Bowman gland cells distinguishes both $\mathrm{AD}$ dementia and $\mathrm{MCI}$ due to $\mathrm{AD}$ from cognitively unaffected controls and MCI caused by other diseases than AD. Furthermore, the BSC4090 ratio correlates with the CSF concentrations of phosphorylated tau (ptau181), a marker of AD-related tau pathology. Thus, BSC4090 staining of olfactory mucosa biopsies could be a potential biomarker for the detection of tau in prodromal and early $\mathrm{AD}$ and probably even in preclinical disease stages, especially as the procedure is cost-effective and safe. We did not observe any serious side effects and olfactory mucosa is easily accessible by transnasal biopsy in an outpatient setting within an approximately 30 min time period. Analysis of BSC 4090 ratios resulted in the AUC values comparable to that of established CSF biomarkers and both sensitivity and specificity of BSC4090 could be further optimized by chemical modification. Importantly, BSC4090 labeling was already positive in the early disease stages.

Several previous studies support the notion that olfactory mucosa may reflect pathology of the central nervous system, e.g., in Parkinson's disease, schizophrenia, and rodent tauopathy models [26, 31, 39, 42]. Interestingly, Moon et al. found higher levels of miRNA-206 in olfactory mucosa in $\mathrm{MCI}$ and even more in manifest $\mathrm{AD}$ as compared to controls, thus supporting the suitability of olfactory mucosa tissue as a possible biomarker [30].

The high proportion of cognitively normal individuals who showed at least some BSC4090 positive OE cells could partially be explained by preclinical AD pathology in the $\mathrm{CN}$ group. Based on a recent meta-analysis including 2914 subjects with normal cognition, Jansen et al. reported a 10-44\% prevalence of AD pathology defined by either positive amyloid- $\beta$ PET imaging or pathological CSF biomarkers in the age group between 50 and 90 years [24]. Autopsy studies revealed even higher rates of AD pathology in cognitively normal individuals, ranging from $10 \%$ to as much as $60 \%$ [29].

One of the limitations of our study is the lack of clinical longitudinal follow-up data to assess potential conversions from MCI to $\mathrm{AD}$ or cognitive decline from $\mathrm{CN}$ to $\mathrm{MCI}$ and/or AD. As clinical follow-up data were not available in all the cases and the maximum follow-up interval was 2 years, we had to rely on a CSF biomarker-based distinction between MCI due to $\mathrm{AD}$ and $\mathrm{MCI}$ due to other causes [1]. It is likely that longitudinal follow-up data on MCI progression would have resulted in a better specificity of the BSC4090 marker, as would have post-mortem verification of diagnoses. During the 2 year period following this study, two of the six subjects in the $\mathrm{MCI}$ due to $\mathrm{AD}$ group clinically converted to AD dementia. Both showed the initial BSC4090 ratios well above the cut-off value (0.065) with BSC4090 ratios of 0.392 and 0.87 , respectively. It would have been interesting to follow whether other participants in the MCI and $\mathrm{CN}$ groups with BSC4090 ratios above the cut-off value would later on progress towards $\mathrm{AD}$ dementia. Another limitation 
of our study is the small cohort size. Clearly, our results warrant a biomarker-aided, prospective, and longitudinal cohort with a higher number of participants, ideally with post-mortem verification of diagnoses, to further validate our findings.

In OE, the staining pattern of BSC4090 was never fibrillar and also negative for Thioflavin $\mathrm{T}$ which binds to $\beta$-sheet structures present in amyloid- $\beta$ or tau fibrils (data not shown). As our in vitro NMR data suggest that BSC4090 can bind to both, fibrillar and pre-fibrillar tau species, but not to monomeric tau, BSC4090 positive tau in OE is most likely pre-fibrillar. Binding of BSC4090 to pre-fibrillar tau aggregates may also explain why BSC4090 detects very early stages of disease pathology.

In an in vitro assay to test BSC4090 binding affinities to recombinant tau and amyloid- $\beta$ aggregates, the IC50 values for BSC4090 were 4 and $54 \mathrm{nM}$, respectively [13]. This indicates that the methoxy-X04 derivative BSC4090 has a high affinity and selectivity for aggregated tau versus amyloid- $\beta$ fibrils. We observed partial colocalisation of BSC4090 with tau phosphorylated at S202/T205 (AT8) (suppl. Fig. S3). However, we could not detect amyloid- $\beta$ pathology in our OE biopsies as immunostaining with $6 \mathrm{E} 10$ antibody against amino acids $3-8$ of the amyloid- $\beta$ peptide revealed no $6 \mathrm{E} 10$ positive olfactory epithelial cells (data not shown). We cannot rule out that BSC4090 labeling of OE glands is caused by unspecific binding of BSC4090 to other proteins than tau, although, in post-mortem brain, BSC4090 labeling patterns resembled the typical morphology of NFTs and colocalized with AT8 tau staining [13].

\section{Conclusions}

Although the number of subjects in our study is limited and a longitudinal observation of disease progression would have been desirable, we provide the evidence that (1) OE biopsies are a feasible diagnostic approach, (2) BSC4090 positive pathology is present in OE in prodromal and clinical AD and (3) BSC4090 may be applicable to ante mortem diagnosis and even may bear the potential to be employed as a prognostic marker in prodromal and preclinical AD. However, biomarker-aided longitudinal cohorts are required to better evaluate the diagnostic and prognostic potential of BSC4090 and other methoxy-XO derivatives, as well as indepth analysis of potentially unspecific binding sites.

Acknowledgements We are grateful to Angela Dettmar, DZNE Göttingen, for expert technical assistance and to Sabrina Hübschmann, DZNE Bonn, for preparation of recombinant tau proteins. A.S. and M.Z. were supported by grants from the German Research foundation Cluster of Excellence "Nanoscale Microscopy and Molecular Physiology of the Brain" (CNMPB) and the Center for Molecular Physiology of the Brain (CMPB). A.S. received funding by the German Research Foundation
(Deutsche Forschungsgemeinschaft) grants SCHN1265 2-1 and 1-1. B.S. thanks Hans-und-Ilse-Breuerstiftung for support.

Author contributions $\mathrm{HP}, \mathrm{MaC}, \mathrm{MZ}, \mathrm{EM}$, and $\mathrm{AS}$ : conception and design of the study. FI, BW, JT, HK, MC, MK, DR, FL, PW, CB, SH, SR, ES, LT, BS, MZ, MoC, and AS: acquisition and analysis of data. MZ, EM, and AS: drafting the manuscript. All authors read and approved the final manuscript.

\section{Compliance with ethical standards}

Conflict of interest Dr. Schneider received research support from Actelion which was unrelated to the present study. She is a principal investigator in industry-sponsored clinical trials for Merck MSD, Eli Lilly, Biogen and Ionis. Dr. Pellkofer Dr. Ihler, Dr. Weiss, J. Trothe, Dr. Kadavath, M. Chongtham, Dr. Kunadt, Dr. Riedel, F. Lornsen, Dr. Wilken, Dr. Bartels, S. Hirschel, S. Russo, E. Stransky, Dr.Trojan, Dr. Schmidt, Dr. Mandelkow, Dr. Zweckstetter, and Dr. Canis reported no potential conflicts of interest relevant to this manuscript.

\section{References}

1. Albert MS, DeKosky ST, Dickson D, Dubois B, Feldman HH, Fox NC, Gamst A, Holtzman DM, Jagust WJ, Petersen RC, Snyder PJ, Carrillo MC, Thies B, Phelps CH (2011) The diagnosis of mild cognitive impairment due to Alzheimer's disease: recommendations from the National Institute on Aging-Alzheimer's Association Workgroups on Diagnostic Guidelines for Alzheimer's Disease. Alzheimer's Dement 7:270-279

2. Arnold SE, Lee EB, Moberg PJ, Stutzbach L, Kazi H, Han LY, Lee VM, Trojanowski JQ (2010) Olfactory epithelium amyloid-beta and paired helical filament-tau pathology in Alzheimer disease. Ann Neurol 67:462-469

3. Arnold SE, Smutzer GS, Trojanowski JQ, Moberg PJ (1998) Cellular and molecular neuropathology of the olfactory epithelium and central olfactory pathways in Alzheimer's disease and schizophrenia. Ann N Y Acad Sci 855:762-775

4. Attems J, Jellinger KA (2006) Olfactory tau pathology in alzheimer disease and mild cognitive impairment. Clin Neuropathol 25:265-271

5. Barghorn S, Biernat J, Mandelkow E (2005) Purification of recombinant tau protein and preparation of alzheimer-paired helical filaments in vitro. Methods Mol Biol 299:35-51

6. Barresi M, Ciurleo R, Giacoppo S, Foti Cuzzola V, Celi D, Bramanti P, Marino S (2012) Evaluation of olfactory dysfunction in neurodegenerative diseases. J Neurol Sci 323:16-24

7. Bateman RJ, Xiong C, Benzinger TL, Fagan AM, Goate A, Fox NC, Marcus DS, Cairns NJ, Xie X, Blazey TM, Holtzman DM, Santacruz A, Buckles V, Oliver A, Moulder K, Aisen PS, Ghetti B, Klunk WE, McDade E, Martins RN, Masters CL, Mayeux R, Ringman JM, Rossor MN, Schofield PR, Sperling RA, Salloway S, Morris JC, Dominantly Inherited Alzheimer N (2012) Clinical and biomarker changes in dominantly inherited alzheimer's disease. N Engl J Med 367:795-804

8. Bax A, Ikura M, Kay LE, Torchia DA, Tschudin R (1990) Comparison of different modes of 2-dimensional reverse-correlation nmr for the study of proteins. J Magn Reson 86:304-318

9. Bax AIM, Kay LE, Torchia DA, Tschudin R (1990) Comparison of different modes of 2-dimensional reverse-correlation $\mathrm{nmr}$ for the study of proteins. J Magn Reson 86:304-318

10. Becker S, Pflugbeil C, Groger M, Canis M, Ledderose GJ, Kramer MF (2012) Olfactory dysfunction in seasonal and perennial allergic rhinitis. Acta Otolaryngol 132:763-768 
11. Bodenhausen G, Ruben DJ (1980) Natural abundance n-15 $\mathrm{nmr}$ by enhanced heteronuclear spectroscopy. Chem Phys Lett 69:185-189

12. Bolander A, Kieser D, Scholz C, Heyny-von Haussen R, Mall G, Goetschy V, Czech C, Schmidt B (2014) Synthesis of methoxy$\mathrm{x} 04$ derivatives and their evaluation in alzheimer's disease pathology. Neurodegener Dis 13:209-213

13. Bolander A, Kieser D, Voss C, Bauer S, Schon C, Burgold S, Bittner T, Holzer J, Heyny-von Haussen R, Mall G, Goetschy V, Czech C, Knust H, Berger R, Herms J, Hilger I, Schmidt B (2012) Bis(arylvinyl)pyrazines, -pyrimidines, and -pyridazines as imaging agents for tau fibrils and beta-amyloid plaques in Alzheimer's disease models. J Med Chem 55:9170-9180

14. Braak H, Del Trecidi K (2015) Neuroanatomy and pathology of sporadic Alzheimer's disease. Adv Anat Embryol Cell Biol 215:1-162

15. Devanand DP, Lee S, Manly J, Andrews H, Schupf N, Doty RL, Stern Y, Zahodne LB, Louis ED, Mayeux R (2015) Olfactory deficits predict cognitive decline and alzheimer dementia in an urban community. Neurology 84:182-189

16. Doty RL (2017) Olfactory dysfunction in neurodegenerative diseases: is there a common pathological substrate? Lancet Neurol $16: 478-488$

17. Dumurgier J, Laplanche JL, Mouton-Liger F, Lapalus P, Indart S, Prevot M, Peoc'h K, Hugon J, Paquet C (2014) The screening of Alzheimer's patients with CSF biomarkers, modulates the distribution of apoe genotype: Impact on clinical trials. J Neurol 261:1187-1195

18. Ehrensperger MM, Berres M, Taylor KI, Monsch AU (2010) Early detection of Alzheimer's disease with a total score of the german cerad. J Int Neuropsychol Soc (JINS) 16:910-920

19. Fagan AM, Mintun MA, Shah AR, Aldea P, Roe CM, Mach RH, Marcus D, Morris JC, Holtzman DM (2009) Cerebrospinal fluid tau and ptau(181) increase with cortical amyloid deposition in cognitively normal individuals: implications for future clinical trials of Alzheimer's disease. EMBO Mol Med 1:371-380

20. Gustke N, Trinczek B, Biernat J, Mandelkow EM, Mandelkow E (1994) Domains of tau protein and interactions with microtubules. Biochemistry 33:9511-9522

21. Hernandez F, Avila J (2008) Tau aggregates and tau pathology. J Alzheimer's Dis (JAD) 14:449-452

22. Hock C, Golombowski S, Muller-Spahn F, Peschel O, Riederer A, Probst A, Mandelkow E, Unger J (1998) Histological markers in nasal mucosa of patients with Alzheimer's disease. Eur Neurol 40:31-36

23. Jack CR Jr, Knopman DS, Jagust WJ, Petersen RC, Weiner MW, Aisen PS, Shaw LM, Vemuri P, Wiste HJ, Weigand SD, Lesnick TG, Pankratz VS, Donohue MC, Trojanowski JQ (2013) Tracking pathophysiological processes in Alzheimer's disease: an updated hypothetical model of dynamic biomarkers. Lancet Neurol $12: 207-216$

24. Jansen WJ, Ossenkoppele R, Knol DL, Tijms BM, Scheltens P, Verhey FR, Visser PJ, Amyloid Biomarker Study G, Aalten P, Aarsland D, Alcolea D, Alexander M, Almdahl IS, Arnold SE, Baldeiras I, Barthel H, van Berckel BN, Bibeau K, Blennow K, Brooks DJ, van Buchem MA, Camus V, Cavedo E, Chen K, Chetelat G, Cohen AD, Drzezga A, Engelborghs S, Fagan AM, Fladby T, Fleisher AS, van der Flier WM, Ford L, Forster S, Fortea J, Foskett N, Frederiksen KS, Freund-Levi Y, Frisoni GB, Froelich L, Gabryelewicz T, Gill KD, Gkatzima O, Gomez-Tortosa E, Gordon MF, Grimmer T, Hampel H, Hausner L, Hellwig S, Herukka SK, Hildebrandt H, Ishihara L, Ivanoiu A, Jagust WJ, Johannsen P, Kandimalla R, Kapaki E, Klimkowicz-Mrowiec A, Klunk WE, Kohler S, Koglin N, Kornhuber J, Kramberger MG, Van Laere K, Landau SM, Lee DY, de Leon M, Lisetti V, Lleo A, Madsen K, Maier W, Marcusson J, Mattsson N, de Mendonca A,
Meulenbroek O, Meyer PT, Mintun MA, Mok V, Molinuevo JL, Mollergard HM, Morris JC, Mroczko B, Van der Mussele S, Na DL, Newberg A, Nordberg A, Nordlund A, Novak GP, Paraskevas GP, Parnetti L, Perera G, Peters O, Popp J, Prabhakar S, Rabinovici GD, Ramakers IH, Rami L, Resende de Oliveira C, Rinne JO, Rodrigue KM et al (2015) Prevalence of cerebral amyloid pathology in persons without dementia: a meta-analysis. JAMA 313:1924-1938

25. Kadavath H, Hofele RV, Biernat J, Kumar S, Tepper K, Urlaub H, Mandelkow E, Zweckstetter M (2015) Tau stabilizes microtubules by binding at the interface between tubulin heterodimers. Proc Natl Acad Sci USA 112:7501-7506

26. Lavoie J, Sawa A, Ishizuka K (2017) Application of olfactory tissue and its neural progenitors to schizophrenia and psychiatric research. Curr Opin Psychiatry 30:176-183

27. Lee JH, Goedert M, Hill WD, Lee VM, Trojanowski JQ (1993) Tau proteins are abnormally expressed in olfactory epithelium of alzheimer patients and developmentally regulated in human fetal spinal cord. Exp Neurol 121:93-105

28. Mayer M, Meyer B (1999) Characterization of ligand binding by saturation transfer difference nmr spectroscopy. Angew Chem Int Ed 38:1784-1788

29. Montine TJ (2011) Prevalence estimates for latent neurodegenerative disease. Toxicol Pathol 39:99-102

30. Moon J, Lee ST, Kong IG, Byun JI, Sunwoo JS, Shin JW, Shim JY, Park JH, Jeon D, Jung KH, Jung KY, Kim DY, Lee SK, Kim M, Chu K (2016) Early diagnosis of Alzheimer's disease from elevated olfactory mucosal mir-206 level. Sci Rep 6:20364

31. Mor E, Kano S, Colantuoni C, Sawa A, Navon R, Shomron N (2013) Microrna-382 expression is elevated in the olfactory neuroepithelium of schizophrenia patients. Neurobiol Dis 55:1-10

32. Morris JC, Mohs RC, Rogers H, Fillenbaum G, Heyman A (1988) Consortium to establish a registry for Alzheimer's disease (cerad) clinical and neuropsychological assessment of Alzheimer's disease. Psychopharmacol Bull 24:641-652

33. Mukrasch MD, Bibow S, Korukottu J, Jeganathan S, Biernat J, Griesinger C, Mandelkow E, Zweckstetter M (2009) Structural polymorphism of 441-residue tau at single residue resolution. PLoS Biol 7:e34

34. Mukrasch MD, Biernat J, von Bergen M, Griesinger C, Mandelkow E, Zweckstetter M (2005) Sites of tau important for aggregation populate $\{$ beta $\}$-structure and bind to microtubules and polyanions. J Biol Chem 280:24978-24986

35. Ossenkoppele R, Jansen WJ, Rabinovici GD, Knol DL, van der Flier WM, van Berckel BN, Scheltens P, Visser PJ, Amyloid PETSG, Verfaillie SC, Zwan MD, Adriaanse SM, Lammertsma AA, Barkhof F, Jagust WJ, Miller BL, Rosen HJ, Landau SM, Villemagne VL, Rowe CC, Lee DY, Na DL, Seo SW, Sarazin M, Roe CM, Sabri O, Barthel H, Koglin N, Hodges J, Leyton CE, Vandenberghe R, van Laere K, Drzezga A, Forster S, Grimmer T, Sanchez-Juan P, Carril JM, Mok V, Camus V, Klunk WE, Cohen AD, Meyer PT, Hellwig S, Newberg A, Frederiksen KS, Fleisher AS, Mintun MA, Wolk DA, Nordberg A, Rinne JO, Chetelat G, Lleo A, Blesa R, Fortea J, Madsen K, Rodrigue KM, Brooks DJ (2015) Prevalence of amyloid pet positivity in dementia syndromes: a meta-analysis. JAMA 313:1939-1949

36. Tabaton M, Cammarata S, Mancardi GL, Cordone G, Perry G, Loeb C (1991) Abnormal tau-reactive filaments in olfactory mucosa in biopsy specimens of patients with probable Alzheimer's disease. Neurology 41:391-394

37. Talamo BR, Rudel R, Kosik KS, Lee VM, Neff S, Adelman L, Kauer JS (1989) Pathological changes in olfactory neurons in patients with Alzheimer's disease. Nature 337:736-739

38. Thal DR (2015) Clearance of amyloid beta-protein and its role in the spreading of Alzheimer's disease pathology. Front Aging Neurosci 7:25 
39. Van de Bittner GC, Riley MM, Cao L, Ehses J, Herrick SP, Ricq EL, Wey HY, O’Neill MJ, Ahmed Z, Murray TK, Smith JE, Wang C, Schroeder FA, Albers MW, Hooker JM (2017) Nasal neuron pet imaging quantifies neuron generation and degeneration. J Clin Investig 127:681-694

40. Wilson RS, Arnold SE, Schneider JA, Boyle PA, Buchman AS, Bennett DA (2009) Olfactory impairment in presymptomatic Alzheimer's disease. Ann N Y Acad Sci 1170:730-735

41. Winblad B, Palmer K, Kivipelto M, Jelic V, Fratiglioni L, Wahlund LO, Nordberg A, Backman L, Albert M, Almkvist O, Arai H, Basun H, Blennow K, de Leon M, DeCarli C, Erkinjuntti T, Giacobini E, Graff C, Hardy J, Jack C, Jorm A, Ritchie K, van
Duijn C, Visser P, Petersen RC (2004) Mild cognitive impairmentbeyond controversies, towards a consensus: report of the international working group on mild cognitive impairment. J Intern Med 256:240-246

42. Witt M, Bormann K, Gudziol V, Pehlke K, Barth K, Minovi A, Hahner A, Reichmann H, Hummel T (2009) Biopsies of olfactory epithelium in patients with Parkinson's disease. Mov Disord 24:906-914

43. Yamagishi M, Ishizuka Y, Seki K (1994) Pathology of olfactory mucosa in patients with alzheimer's disease. Ann Otol Rhinol Laryngol 103:421-427 